\title{
Interpretation of the cosmic ray positron and electron excesses with an annihilating-decaying dark matter scenario
}

\author{
Lei Feng ${ }^{a}$, Zhaofeng Kang ${ }^{b}$, Qiang Yuan ${ }^{a, c, d}$, Peng-Fei Yin ${ }^{e}$, and Yi-Zhong Fan ${ }^{a, c}$ \\ ${ }^{a}$ Key Laboratory of Dark Matter and Space Astronomy, Purple Mountain Observatory, Chinese \\ Academy of Sciences, Nanjing 210008, China \\ ${ }^{b}$ School of physics, Huazhong University of Science and Technology, Wuhan 430074, China \\ ${ }^{c}$ School of Astronomy and Space Science, University of Science and Technology of China, Hefei \\ 230026, China \\ ${ }^{d}$ Center for High Energy Physics, Peking University, Beijing 100871, China \\ ${ }^{e}$ Key Laboratory of Particle Astrophysics, Institute of High Energy Physics, Chinese Academy of \\ Sciences, Beijing 100049, China \\ E-mail: fenglei@pmo.ac.cn, zhaofengkang@gmail.com, yuanq@pmo.ac.cn, \\ yinpf@ihep.ac.cn, yzfan@pmo.ac.cn
}

ABSTRACT: The precise measurements of energy spectra of cosmic ray positrons and/or electrons by recent experiments show clear excesses above $10 \mathrm{GeV}$. Moreover, a potential sharp spectral feature was suggested by the Dark Matter Particle Explorer (DAMPE) data. These results inspire quite a number of discussions on the connection with either the annihilation/decay of dark matter (DM) or the astrophysical origins. Here we discuss a DM scenario in which DM particles could annihilate and decay into standard model particle pairs simultaneously. In this model, the peak structure is due to the DM annihilation in a nearby subhalo and the broad positron/electron excesses are due to the decay of DM in the Milky Way. This model can reasonably explain the DAMPE and AMS-02 data of the total $e^{+} e^{-}$spectra and the positron fraction, with model parameters being consistent with existing constraints. A simple realization of such a DM model is the spin-1 vector DM model.

KEYWORDS: dark matter, cosmic rays

ArXiv EPrint: 1902.09235 


\section{Contents}

1 Introduction 1

2 CREs from ADDM 2

2.1 Cosmic ray propagation 2

2.2 DM distribution 3

2.3 Results 4

$\begin{array}{llr}3 & \text { Model } & 6\end{array}$

$\begin{array}{llr}4 & \text { Conclusion } & 9\end{array}$

\section{Introduction}

Dark matter (DM) particles may annihilate or/and decay into standard model particles such as pairs of electrons/positrons and protons/anti-protons, and can hence give rise to excesses in the cosmic ray spectra. Identification of such excesses is one of the most important goals of the indirect detection experiments of DM such as PAMELA [1], ATIC [2], Fermi-LAT [3, 4], and AMS-02 [5, 6]. Some progresses have been made in recent years. The most widely-known phenomena are perhaps the spectral anomalies (excesses) of positrons and the electron plus positron spectra. Either astrophysical source(s)/process(es) [7-14] or the DM annihilation/decay [15-29]) were proposed to account for the data.

The DArk Matter Particle Explorer (DAMPE, [30, 31]), launched on December 17, 2015, is a high-energy particle detector dedicated to DM indirect detection and cosmic ray physics. The DAMPE collaboration reported the precise measurement of the cosmic ray $e^{+}+e^{-}$(CREs) spectrum from $25 \mathrm{GeV}$ to $4.6 \mathrm{TeV}$ [32]. The DAMPE data confirms the spectral hardening of CREs around $50 \mathrm{GeV}$ [4], and reveals clearly a spectral softening at $\sim 0.9 \mathrm{TeV}[32]$. These results are consistent with previously reported CRE excesses $[2-4,6]$. Moreover, there might be a sharp peak at $\sim 1.4 \mathrm{TeV}$. Although the significance of the current data is relatively low $(\sim 2.3 \sigma$ considering the look-elsewhere effect [33]), this structure, if confirmed, should imply the existence of nearby quasi-monoenergetic electron sources [34].

If the DM annihilation or decay is employed to account for the peak, the DM particles should annihilate or decay dominantly into leptons, and the annihilation or decay should occur in local regions not far away from the solar system. This is because that TeV CREs lose their energies very quickly when travelling in the Milky Way, and can not reach us if they were generated far away. The quark channels should be dramatically suppressed due to the constraints of anti-protons. Such models have been proposed in literature [35-71]. See Ref. [72] for a special review of relevant studies. 
The main purpose of this work is to interpret both the sub- $\mathrm{TeV}$ positron and electron excesses and the DAMPE peak simultaneously in an annihilating plus decaying DM (ADDM) model. It is natural to speculate that DM particles with a limited lifetime can at the same time annihilate with each other. While the annihilation products, presumed to be leptons, can explain the peak excess around $1.4 \mathrm{TeV}$, the decay products with lower energies can account for the sub-TeV electron/positron excesses. We will investigate whether such a scenario can be realized to account for the data, without violating existing constraints from e.g., $\gamma$-ray observations [73-76] and the cosmic microwave background (CMB) data [77].

This paper is organized as follows. In Section II, we compare ADDM model prediction with the AMS-02 positron fraction and the AMS-02/DAMPE CRE data. In Section III, we discuss a possible theoretical realization of such an ADDM scenario. Our conclusions are summarized in Section IV.

\section{CREs from ADDM}

\subsection{Cosmic ray propagation}

The general form of cosmic ray propagation equation reads [78]:

$$
\begin{aligned}
\frac{\partial \psi}{\partial t} & =q(\mathbf{r}, p)+\nabla \cdot\left(D_{x x} \nabla \psi-\mathbf{V} \psi\right)+\frac{\partial}{\partial p} p^{2} D_{p p} \frac{\partial}{\partial p} \frac{\psi}{p^{2}} \\
& -\frac{\partial}{\partial p}\left[\dot{p} \psi-\frac{p}{3}(\nabla \cdot \mathbf{V}) \psi\right]-\frac{\psi}{\tau_{f}}-\frac{\psi}{\tau_{r}}
\end{aligned}
$$

where $\psi=\psi(\mathbf{r}, p, t)$ is the phase space density, $q(\mathbf{r}, p)$ denotes the source function, $D_{x x}=$ $\beta D_{0}(E / 4 \mathrm{GeV})^{\delta}$ is the spatial diffusion coefficient, $\mathbf{V}=d V / d z \cdot \mathbf{z}$ represents the convection velocity, $D_{p p}$ denotes the diffusion coefficient in the momentum space which is characterized by the Alfvenic speed $v_{A}$ and is to describe the reacceleration of particles in the interstellar medium, $\dot{p}=d p / d t$ is the momentum loss rate, and $\tau_{f}\left(\tau_{r}\right)$ is the time scale of the fragmentation (the radioactive decay).

Typically the Boron-to-Carbon ratio, the radioactive secondary abundances, as well as diffuse $\gamma$-rays are used to constrain the cosmic ray propagation parameters [78-81]. In this work, we consider a few propagation model configurations and parameter settings, which are summarized in Table 1. For the diffusion-reacceleration configuration, we also adopt several values of the halo height following Ref. [82]. As a benchmark setting, we adopt the diffusion-reacceleration model with a half-height of the propagation cynlinder $z_{h}=4 \mathrm{kpc}$, as shown in bold in Table 1 (model II). Different propagation models affect mainly the lowenergy spectra of electrons and positrons, and have very minor effects on the DM model which is the focus of this work. This is because the main propagation effect of high-energy CREs is radiative cooling rather than the diffusion, convection, and reacceleration. Highenergy CREs can only propagate a very limited distance in the Galaxy before they get cooled down. As an illustration, the cooling time for TeV CREs is about $3 \times 10^{5}$ years and the corresponding diffusion length is about $1 \mathrm{kpc}$ for the diffusion parameter setting II. 
Table 1. Propagation parameters. The benchmark setting is model II which is shown in bold.

\begin{tabular}{cccccc}
\hline \hline Model & $\begin{array}{c}D_{0}^{a} \\
\left(10^{28} \mathrm{~cm}^{2} \mathrm{~s}^{-1}\right)\end{array}$ & $\begin{array}{c}z_{h} \\
(\mathrm{kpc})\end{array}$ & $\begin{array}{c}v_{A} \\
\left(\mathrm{~km} \mathrm{~s}^{-1}\right)\end{array}$ & $\begin{array}{c}d V_{c} / d z \\
\left(\mathrm{~km} \mathrm{~s}^{-1} \mathrm{kpc}^{-1}\right)\end{array}$ & $\delta$ \\
\hline I & 2.7 & 2.0 & 35.0 & 0 & 0.33 \\
II & 5.3 & 4.0 & 33.5 & 0 & 0.33 \\
III & 9.4 & 10.0 & 28.6 & 0 & 0.33 \\
IV & 4.2 & 10.9 & 0 & 5.4 & 0.59 \\
\hline \hline
\end{tabular}

${ }^{a}$ Diffusion coefficient at rigidity of $4 \mathrm{GV}$.

This propagation equation can be solved numerically, by e.g., GALPROP [83] and DRAGON [84]. In this work we use the LikeDM package [85], which tabulates the outputs from GALPROP and enables fast computation, to calculate the propagation of CREs for all the background (non-DM) components and the Milky Way DM annihilation/decay component of CREs. Note that we will also deal with the CRE propagation from a nearby DM subhalo. For this particular case, we simplify the propagation equation, keeping only the diffusion and energy loss terms, and adopt the analytical Green's function to solve the CRE propagation in a spherically symmetric geometry with infinite boundary conditions [86].

\subsection{DM distribution}

For the annihilation process, the CRE source function is

$$
q(E, r)=\frac{\langle\sigma v\rangle}{2 m_{\mathrm{DM}}^{2}} \frac{\mathrm{d} N}{\mathrm{~d} E} \times \rho^{2}(r),
$$

where $m_{\mathrm{DM}}$ is the mass of the DM particle and $\mathrm{d} N / \mathrm{d} E$ is the spectrum of CREs per annihilation. For the decaying case, the source term is

$$
q(E, r)=\frac{\rho(r)}{m_{\mathrm{DM}} \tau} \frac{\mathrm{d} N}{\mathrm{~d} E},
$$

where $\tau$ is the lifetime of the DM particle.

The DM distribution $\rho(r)$ in the Milky Way is assumed to be an isothermal distribution $[87]$

$$
\rho_{\mathrm{mw}}(r)=\frac{\rho_{s}}{1+\left(r / r_{s}\right)^{2}},
$$

where $\rho_{s}=1.16 \mathrm{GeV} \mathrm{cm}^{-3}$ denotes the finite central density and $r_{s}=5 \mathrm{kpc}$ represents the core radius. As for the subhalo, we assume an NFW distribution [88]

$$
\rho_{\text {sub }}(r)=\frac{\delta_{c} \rho_{\text {crit }}}{\left(r / r_{\mathrm{s}}\right)\left(1+r / r_{\mathrm{s}}\right)^{2}}
$$

where $r_{s}$ and $\delta_{c}$ are the scale radius and characteristic density, $\rho_{\text {crit }}=3 H^{2} / 8 \pi G$ is the critical density of the Universe. The $\delta_{c}$ parameter relates with the subhalo concentration parameter $c=r_{v} / r_{s}$, where $r_{v}$ is the virial radius, as [89]

$$
\delta_{c}=7.213 \delta_{V}=\frac{200}{3} \frac{c^{3}}{\ln (1+c)-c /(1+c)} .
$$


For subhalos in the solar neighborhood, we have approximately [89]

$$
\delta_{V}=1.2 \times 10^{6}\left(\frac{M_{\mathrm{sub}}}{10^{6} M_{\odot}}\right)^{-0.18},
$$

where $M_{\text {sub }}$ is the mass of the subhalo. The tidal force of the Milky Way DM halo would remove the DM beyond a so-called tidal radius from the subhalo. Adopting the method of Ref. [89], the tidal radius is found to be roughly 0.2 times of the original virial radius of a subhalo [34]. Therefore, the DM distribution of the subhalo is an NFW distribution truncated at the tidal radius $r_{t}$.

\subsection{Results}

The background electrons include primary electrons accelerated from conventional cosmic ray sources, whose injection spectrum is parameterized as a three-segment broken powerlaw with an exponential cutoff [34], and secondary electrons from inelastic collisions of cosmic ray nuclei and the interstellar medium. The background electron spectral parameters are tuned to match the data. The background positrons are mainly from the inelastic collisions between cosmic ray nuclei and the medium. The detailed parameters of the CR electron and positron backgrounds are presented in Table 3 in the Appendix. The annihilation and decay contributions from DM in both the Milky Way halo and the nearby subhalo are also added to the model.
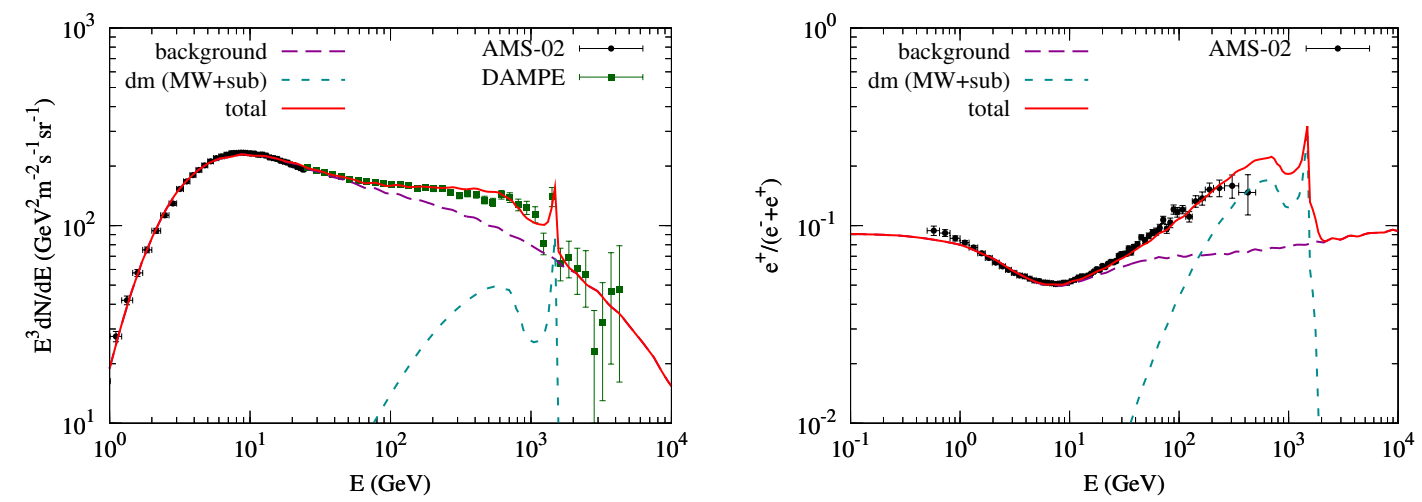

Figure 1. The total CRE fluxes (left) and positron fraction (right) for the ADDM model for propagation model II, compared with the AMS-02 [5,6] and DAMPE [32] data. Here the DM particles annihilate and decay into $e^{+} e^{-}$and $\mu^{+} \mu^{-}$with branching ratios of $1: 1$. Other model parameters are, $m_{\mathrm{DM}}=1.5 \mathrm{TeV},\langle\sigma v\rangle=2.8 \times 10^{-24} \mathrm{~cm}^{3} \mathrm{~s}^{-1}, \tau=9 \times 10^{26} \mathrm{~s}, M_{\mathrm{sub}}=5 \times 10^{4} \mathrm{M}_{\odot}$, and $d=0.1 \mathrm{kpc}$.

Here we take the propagation model setting II as an example to discuss the fitting results. Figure 1 shows the CRE spectrum (left panel) and the positron fraction (right panel) from the model prediction for the propagation model setting II, compared with the measurements $[5,6,32]$. Here the ADDM model parameters are: the mass of the DM particle is $m_{\mathrm{DM}}=1.5 \mathrm{TeV}$, the annihilation cross section is $\langle\sigma v\rangle=2.8 \times 10^{-24} \mathrm{~cm}^{3} \mathrm{~s}^{-1}$, the decay lifetime is $\tau=9 \times 10^{26} \mathrm{~s}$, and the branching ratios are $e: \mu=1: 1$. The subhalo 

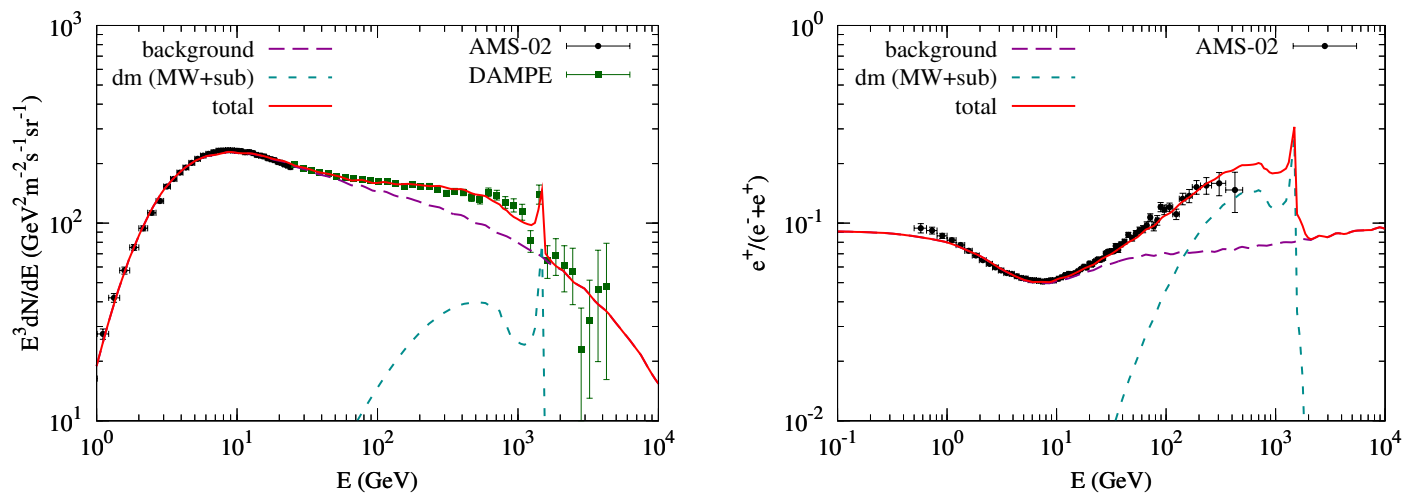

Figure 2. Same as Fig. 1 but for branching ratio of $e: \mu=1: 3$. The mass of subhalo is about $10^{5} \mathrm{M}_{\odot}$. The other parameters are the same as those in Fig. 1.

mass is $M_{\text {sub }}=5 \times 10^{4} \mathrm{M}_{\odot}$, and the distance to the subhalo center is $d=0.1 \mathrm{kpc}$. Figure 2 shows a slightly improved fitting with annihilation/decay branching ratios $e: \mu=1: 3$. It is shown that the model prediction matches well with the data. The parameter values for the other propagation models are compiled in Table 2, which show minor variations among different models. For reference, the comparisons between the model and data for the other three propagations models are given in Figure 5 in the Appendix.

Table 2. The ADDM model parameters and required subhalo masses for different propagation parameters. The DM annihilation/decay branching ratios are assumed to be $e: \mu=1: 1$.

\begin{tabular}{ccccc}
\hline \hline Model & $\begin{array}{c}m_{\mathrm{DM}} \\
(\mathrm{TeV})\end{array}$ & $\begin{array}{c}\langle\sigma v\rangle \\
\left(10^{-24} \mathrm{~cm}^{3} \mathrm{~s}^{-1}\right)\end{array}$ & $\begin{array}{c}\tau \\
\left(10^{26} \mathrm{~s}\right)\end{array}$ & $\begin{array}{c}M_{\text {sub }} \\
\left(10^{4} \mathrm{M}_{\odot}\right)\end{array}$ \\
\hline I & 1.5 & 2.8 & 8.1 & 3.5 \\
II & 1.5 & 2.8 & 9.0 & 5.0 \\
III & 1.5 & 2.8 & 11.3 & 8.0 \\
IV & 1.5 & 2.8 & 9.0 & 4.5 \\
\hline \hline
\end{tabular}

The spike structure around $1.4 \mathrm{TeV}$ is due to the annihilation of $\mathrm{DM}$ in the subhalo, and the sub-TeV broad excesses of positrons and CREs are mainly due to the decay of DM in the Milky Way halo. To clearly see this, we plot in Figure 3 the CRE fluxes from the DM annihilation or decay in either the Milky Way or the subhalo separately. For the parameters we adopt, the subhalo contribution is dominated by the DM annihilation, which gives rises to the $1.4 \mathrm{TeV}$ peak shown in the data. For the Milky Way components, the decay component is slightly larger than the annihilation one. Note that here we need a relatively high contribution from the DM annihilation in the Milky Way (and hence a relatively large cross section), otherwise the $\mathrm{CRE}$ data from $700 \mathrm{GeV}$ to $\mathrm{TeV}$ cannot be well reproduced.

The annihilation cross section and decaying lifetime are marginally consistent with the constraints from $\gamma$-rays [73-76] and CMB [77]. As shown in Ref. [34], the upper limits of the annihilation cross section from CMB are about $3 \times 10^{-24} \mathrm{~cm}^{3} \mathrm{~s}^{-1}$ for the $\mu^{+} \mu^{-}$ 
channel and $10^{-24} \mathrm{~cm}^{3} \mathrm{~s}^{-1}$ for the $e^{+} e^{-}$channel. The lower limits on the decay lifetime from the extragalactic diffuse $\gamma$-ray background are about $4 \times 10^{26}$ s for the $\mu^{+} \mu^{-}$channel and $10^{27} \mathrm{~s}$ for the $e^{+} e^{-}$channel. Therefore, the model parameters derived in this work are not excluded by the current data.

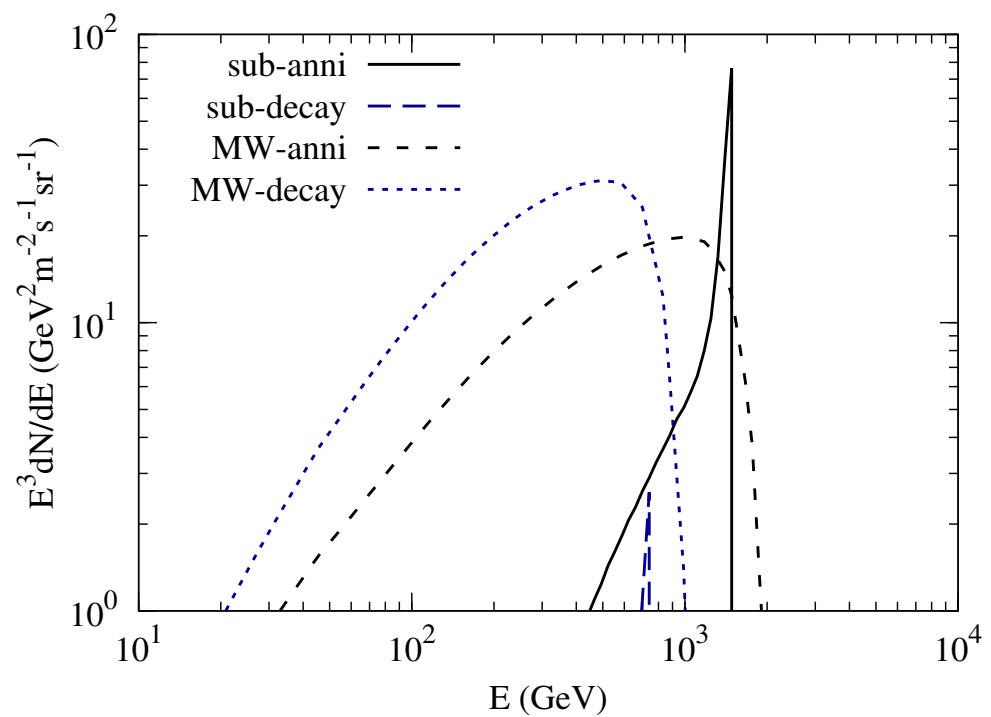

Figure 3. Contributions of the subhalo and MW components to the CRE fluxes for the annihilation and decay modes, respectively. The DM model parameters are the same as those in Figure 1.

The DM annihilation and decay in a nearby subhalo should also be constrained by $\gamma$ rays and CRE anisotropies [34, 90]. In addition, the profile of the line-like feature can also constrain the parameters of the subhalo $[47,90]$. A large distance of subhalo $(\gtrsim 0.3 \mathrm{kpc})$ is ruled out by the width of DAMPE CRE excess, and the halo size is bounded by the Fermi $\gamma$-ray observations [90]. In a forthcoming work, we will show that the $\gamma$-ray emission from this postulated DM subhalo is consistent with the current Fermi data at least in some low-latitude sky regions [91]. Finally, we comment that the probablity of finding such a massive subhalo within a short distance from the Earth is relatively low [34, 92]. According to Fig. 8 of Ref. [34], we estimate that such a probability is about $0.1 \%$ for $d=0.1 \mathrm{kpc}$ and $M_{\text {sub }}=5 \times 10^{4} \mathrm{M}_{\odot}$.

\section{Model}

According to the results in the previous section, we need a primary spectrum which includes contributions from both annihilation and decay of DM into a pair of light charged leptons (referring to $e$ and $\mu$, which are commonly denoted as $\ell$ hereafter). The simplest candidate is supposed to be a spin-0 particle $S$. However, this scenario does not work naturally. We first consider that $S$ annihilates into $\bar{\ell}_{i} \ell_{j}$ via a $t$-channel charged fermionic mediator. To avoid the $p$-wave suppression, it requires a large chiral violation which at the same time gives rise to either a large lepton flavor violation $\bar{\ell}_{i} \rightarrow \ell_{j}+\gamma$ or a large $g_{\ell}-2$; both have been ruled out. Then we consider the $s$-channel annihilation exchanging a spin-0 or 
spin-1 mediator $X$. The latter is again $p$-wave suppressed, whereas the former is not well motivated.

A spin-1 vector DM (VDM) $V_{\mu}$ may works. The reason is that its annihilation into $\bar{\ell} \ell$ is not $p$-wave suppressed even in the absence of chiral violation. In this work we are not aiming at constructing a complete model. We just consider the following minimal $Z_{2}$-invariant effective model

$$
-\mathcal{L}_{1}=\left(\frac{1}{2} m_{V} V^{2}+m_{F} \bar{F} F\right)+\left(g_{V} V_{\mu} \bar{\ell} \gamma^{\mu} P_{L} F+h . c .\right),
$$

where the $Z_{2}$-odd fermion $F$ is a Dirac fermion, mediating the $t$-channel annihilation $V V \rightarrow$ $\bar{\ell} \ell$. Here the VDM is leptophilic because it couples dominantly to light leptons. To suppress the lepton flavor violation, we further require that each lepton flavor $\ell_{i}$ has its own partner $F_{i}$, and they do not give rise to new flavor violation. Different from the scalar DM case, the annihilation cross section has unsuppressed $s$-wave contribution

$$
\left\langle\sigma_{\bar{\ell} \ell} v\right\rangle=\frac{g_{V}^{4}}{9 \pi} \frac{1}{m_{V}^{2}} \frac{r^{4}}{\left(r^{2}+1\right)^{2}},
$$

with $r \equiv m_{V} / m_{F}<1$. Without chiral violation, the contribution to $g_{\ell}-2$ from Eq. (3.1) is not significant, given by [93]

$$
g_{\ell}-2=-\left|g_{V}\right|^{2} \frac{m_{\ell}^{2}}{m_{V}^{2}} \frac{5-14 r^{2}+39 r^{4}-38 r^{6}+8 r^{8}+18 r^{4} \ln r^{2}}{96 \pi^{2}\left(1-r^{2}\right)^{4}},
$$

which is suppressed by the light fermion mass square. We display the numerical results of Eq. (3.2) and Eq. (3.3) in Fig. 4. We find that for $\left\langle\sigma_{\bar{\ell} \ell} v\right\rangle$ as large as $\sim 100$ pb, the resulting $g_{\ell}-2$ can still lie below the current uncertainties, which are $\sim 10^{-12}$ and $10^{-9}$ for electrons and muons, respectively. The conclusion is particularly true in the region with $r \sim 1$. Note that for an annihilation cross section as large as $100 \mathrm{pb}$, the relic density after freezing-out will be too small to account for the total DM budget. One way to overcome this problem is assuming that the early universe after reheating does not reach a very high temperature, but much below the TeV scale. Consequently, the VDM and as well the mediator $F$ never reach full thermal equilibrium, so one can not calculate VDM relic density via the usual freeze-out dynamics. Instead, one should track all productions of DM via the scattering like $\ell \bar{\ell} \rightarrow V V$, namely through freeze-in [94]

It is seen that $g_{V} \sim 4$ is needed to make $\left\langle\sigma_{\ell \bar{\ell}} v\right\rangle \sim 100 \mathrm{pb}$, so one should worry if such a VDM is subjected to a strong constraint by direct detection. Since this VDM is leptonic and moreover self-conjugate (a real vector), its leading interaction with the quark electromagnetic current is via the anapole momentum of VDM, described by the dimension- 6 operator [93]

$$
\mathcal{O}_{V}=g_{5} \frac{e}{4 \pi} \frac{\alpha_{V}}{m_{F}^{2}} \partial_{\mu} V^{\alpha} \partial_{\alpha} V_{\nu} F_{\rho \sigma} \epsilon^{\mu \nu \rho \sigma},
$$

with $F_{\rho \sigma}$ the Maxwell field strength tensor. The operator coefficient is estimated from the one-loop diagram with a charged loop, giving $\alpha_{V}=g_{V}^{2} / 4 \pi \sim 1$ and $g_{5} \sim \mathcal{O}(1)$. 

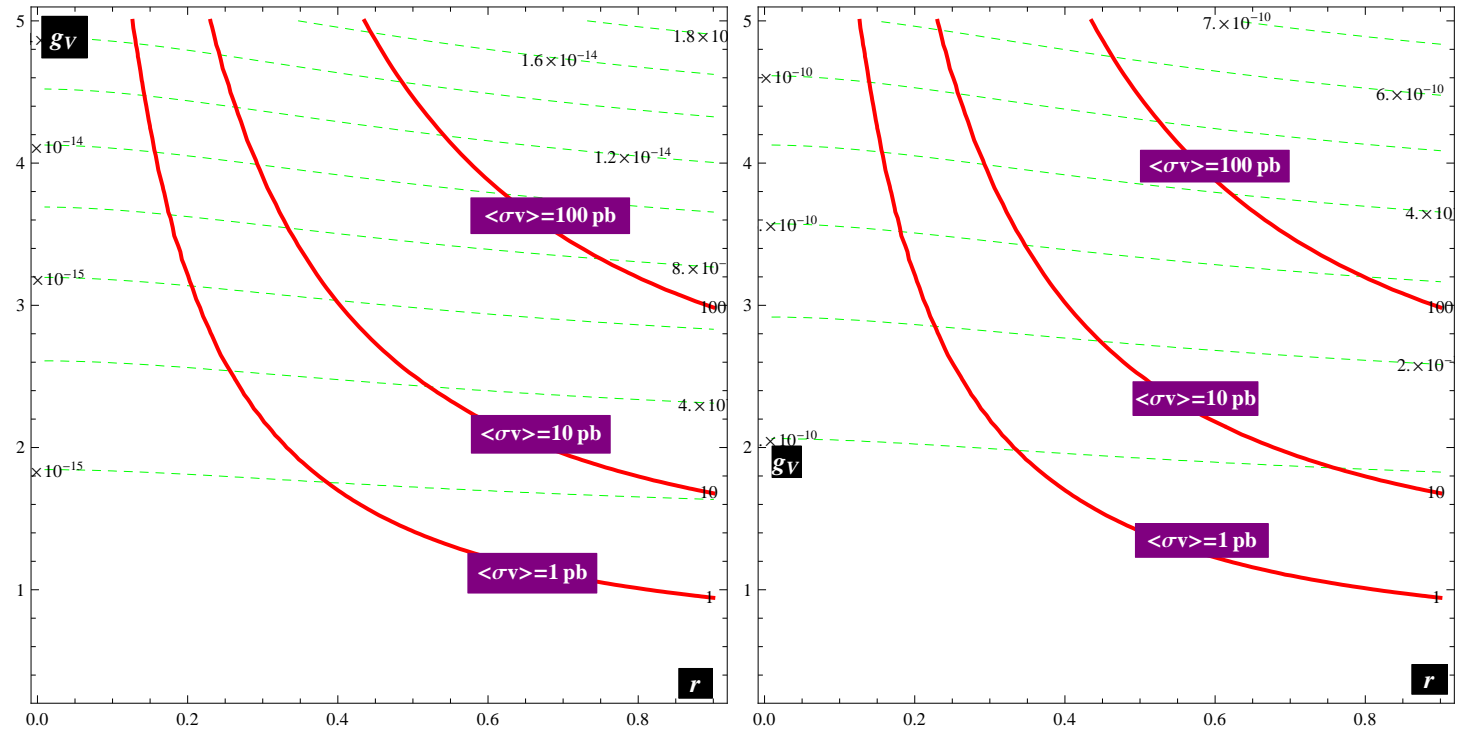

Figure 4. Contours of the annihilation cross section times relative velocity (red thick lines) and the contribution to $g_{\ell}-2$ (green dashed lines) in the $r-g_{V}$ plane. The left panel shows the results for $g_{e}-2$, and the right panel shows those for $g_{\mu}-2 . m_{V}=1.5 \mathrm{TeV}$ is adopted.

The resulting VDM-nucleon scattering is suppressed by $p$-wave in the nonrelativistic limit, which is explicit in the following estimation on the cross section ${ }^{1}$,

$$
\sigma_{p} \sim 0.5 \times 10^{-13}\left(\frac{1.5 \mathrm{TeV}}{m_{F}}\right)^{4}\left(\frac{\alpha_{V}}{1.0}\right)^{2}\left(\frac{v}{10^{-3}}\right)^{2} \mathrm{pb} .
$$

It is four orders of magnitude below the current strongest bound from XENON1T. Actually, it already goes beneath the neutrino floor and therefore probably can not be probed in the direct detection experiments

Actually, a structure similar to Eq. (3.1) is presented in the little Higgs model with $T$-parity [96] or the extra dimensional theory with KK-pairty [97, 98], where the lightest $T /$ KK-odd vector boson is the VDM candidate. In these models all left-handed fermions are accompanied with $T /$ KK-odd heavy fermions. But after identifying $g_{V}$ with the $U(1)_{Y}$ gauge coupling $g^{\prime}$, the cross section is far from the required value to explain the CRE data. From the required large $g_{V}$ value, it is of interest to build VDM in the context of the composite Higgs model.

Now we discuss the VDM decay. To make it decay into a pair of light leptons, the simplest approach is to introduce a term which breaks $Z_{2}$ explicitly as the following:

$$
-\mathcal{L}_{\text {decay }}=\epsilon_{V} V_{\mu} \bar{\ell} \gamma^{\mu} \ell
$$

The resulting lifetime of $V$ is $\tau_{V} \sim 10^{26} \mathrm{~s}\left(10^{-26} / \epsilon_{V}\right)^{2}\left(1.5 \mathrm{TeV} / m_{V}\right)$. To understand the smallness of $\epsilon_{V}$, one needs the completion of the effective model and specifies the origin of

\footnotetext{
${ }^{1}$ We did not make a solid calculation, but borrowed the result from a similar model studied in Ref. [95], based on the Majorana fermion anapole dark matter.
} 
$Z_{2}$. For instance, it might be due to a spontaneous but tiny break of $Z_{2}$ by a scalar field; such a break results in a tiny mixing between $V$ and some leptophilic gauge bosons, then giving rise to the effective operator in Eq. (3.6) through this tiny mixing. Alternatively, if $Z_{2}$ is identified with the $T$-parity, its violation may be due to anomaly [99].

To end up this section we make a comment on the spin- $1 / 2$ candidate $\chi$. It may also give the desired CRE spectrum if its interaction is largely specified by a spin- 1 intermediate state $X_{\mu}$ which is merely slightly lighter than $\chi$ and dominantly couples to $\ell^{2}$. In such a case, the DM cascade decay $\chi \rightarrow \nu+X(\rightarrow \bar{\ell} \ell)$ produces the $e^{+} e^{-}$spectrum similar to that from the two-body decay. Moreover, the annihilation $\bar{\chi}+\chi \rightarrow \bar{\ell}_{i}+\ell_{j}$ is not $p$-wave suppressed. We leave this scenario for future studies.

\section{Conclusion}

In this work, we propose an ADDM model to interpret the positron and CRE data from AMS-02 and DAMPE. In this model we assume that DM particles could annihilate and decay into light leptons (electrons and muons) at the same time. It is shown that the annihilation in a nearby subhalo can explain the potential peak structure of the CRE spectrum, and the decay in the Milky Way halo can explain the broad sub-TeV excesses of both positrons and CREs. The model parameters, although somehow tuned to explain the data, are consistent with the current $\gamma$-ray and CMB constraints.

A spin-1 VDM was proposed as a particle realization of the model. The annihilation into leptons of the VDM is not $p$-wave suppressed even in the absence of chiral violation. At the same time, its contribution to $g-2$ is not significant.

\section{Acknowledgments}

We thank Dr. Ran Ding for helpful discussions and suggestions. This work was supported in part by the National Key Research and Development Program of China (No. 2016YFA0400200), the National Natural Science Foundation of China (Nos. 11773075, 11525313, 11722328, U1738210, U1738209, U1738206) and the Youth Innovation Promotion Association of Chinese Academy of Sciences (No. 2016288). QY is also supported by the 100 Talents Program of Chinese Academy of Sciences.

\section{Appendix: results for the other cosmic ray propagation parameters}

The injection spectrum of the background electrons is parameterized as a three-piece broken power-law with an exponential cutoff form, with spectral indices $\nu_{1}, \nu_{2}, \nu_{3}$, break rigidities $R_{\mathrm{br}, 1}, R_{\mathrm{br}, 2}$, and cutoff rigidity $R_{\mathrm{cut}}$. The background positron spectrum is calculated according to the interaction between CR nuclei and the interstellar medium [79]. A renormalization factor $\left(c_{e^{+}}\right)$has been multiplied to the secondary positron spectrum in order to fit the low-energy positron fraction and CRE spectra simultaneously. We further apply a force-field solar modulation model on the propagated electron and positron spectra before comparing with the measurements carried out on top of the atmosphere [101].

\footnotetext{
${ }^{2}$ The $t$-channel annihilation again suffers from the $p$-wave suppression as the scalar case [100].
} 
Table 3. The injection spectral parameters of the background electrons, the renormalization factor of the background positrons, and the force-field potential of the solar modulation.

\begin{tabular}{ccccccccc}
\hline \hline Model & $\nu_{1}$ & $\nu_{2}$ & $\nu_{3}$ & $\begin{array}{c}R_{\mathrm{br}, 1} \\
(\mathrm{GV})\end{array}$ & $\begin{array}{c}R_{\mathrm{br}, 2} \\
(\mathrm{GV})\end{array}$ & $\begin{array}{c}R_{\text {cut }} \\
(\mathrm{GV})\end{array}$ & $c_{e^{+}}$ & $\begin{array}{c}\Phi \\
(\mathrm{GV})\end{array}$ \\
\hline I & 1.48 & 3.11 & 2.61 & 2.2 & 41.0 & $4.0 \times 10^{4}$ & 2.72 & 1.83 \\
II & 1.48 & 3.00 & 2.56 & 2.3 & 55.0 & $1.1 \times 10^{4}$ & 3.27 & 1.42 \\
III & 1.53 & 2.93 & 2.46 & 2.7 & 79.6 & $4.4 \times 10^{3}$ & 3.84 & 1.15 \\
IV & 1.63 & 2.90 & 2.48 & 3.6 & 46.0 & $2.3 \times 10^{4}$ & 2.80 & 0.88 \\
\hline \hline
\end{tabular}
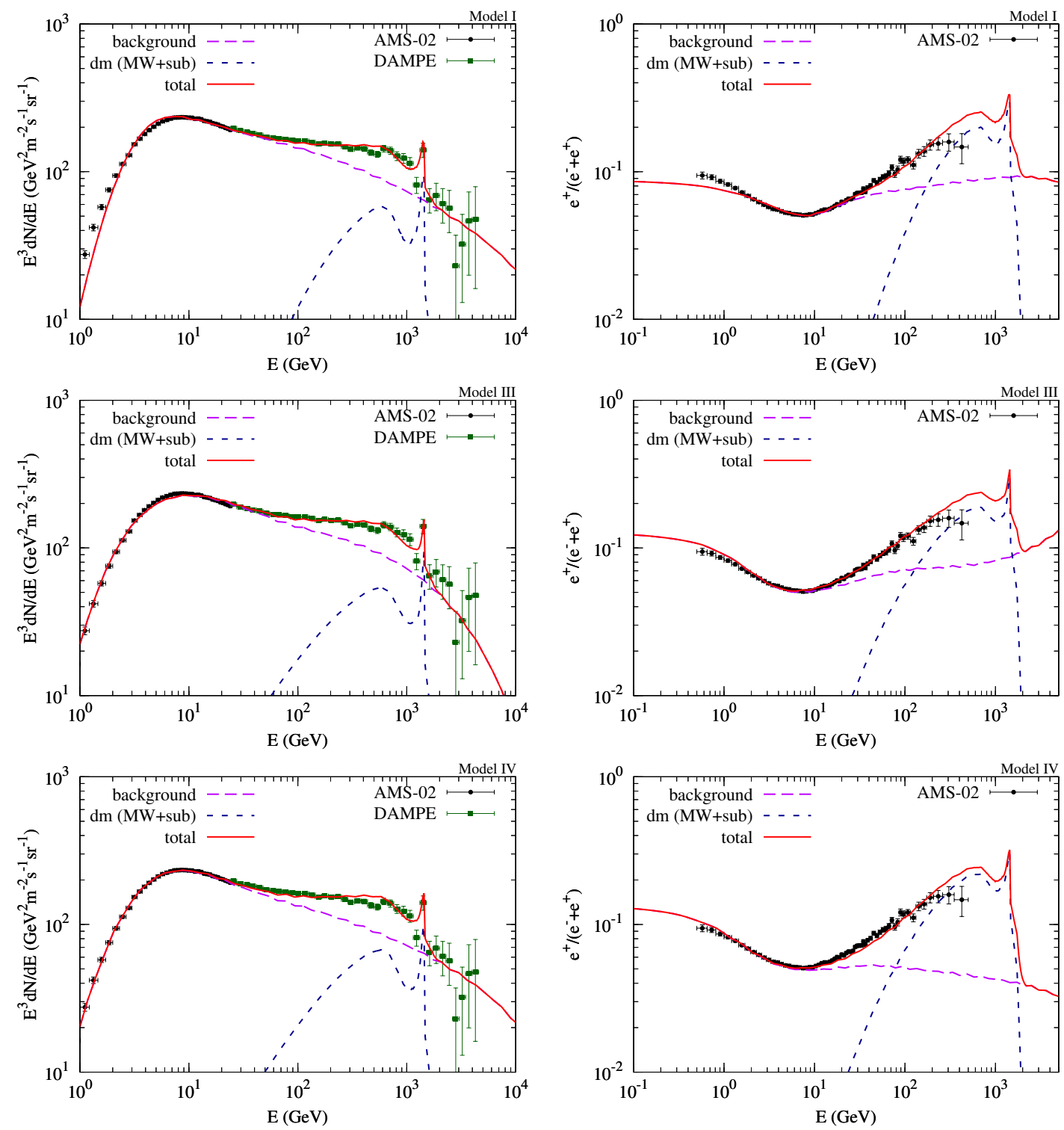

Figure 5. Same as Figure 1 but for cosmic ray propagation parameter settings I (top panels), III (middle panels), and IV (bottom panels). 
The comparisons between the model and data for the other three propagations models are given in Figure 5 for DM annihilation/decay branching ratios $e: \mu=1: 1$. From the figure, we can see that these three propagation models match with the data very well. Similar to CR propagation model II, the annihilation cross section and decaying lifetime as shown in in Table 2 are all consistent with the current $\gamma$-rays [73-76] and CMB [77] observations.

\section{References}

[1] O. Adriani et al. [PAMELA Collaboration], An anomalous positron abundance in cosmic rays with energies 1.5-100 GeV, Nature 458, 607 (2009) [arXiv:0810.4995 [astro-ph]].

[2] J. Chang et al., An excess of cosmic ray electrons at energies of 300-800 GeV, Nature 456, 362 (2008).

[3] A. A. Abdo et al. [Fermi-LAT Collaboration], Measurement of the Cosmic Ray e+ plus espectrum from $20 \mathrm{GeV}$ to $1 \mathrm{TeV}$ with the Fermi Large Area Telescope, Phys. Rev. Lett. 102, 181101 (2009) [arXiv:0905.0025 [astro-ph.HE]].

[4] S. Abdollahi et al. [Fermi-LAT Collaboration], Cosmic-ray electron-positron spectrum from 7 GeV to $2 \mathrm{TeV}$ with the Fermi Large Area Telescope, Phys. Rev. D 95, no. 8, 082007 (2017) [arXiv:1704.07195 [astro-ph.HE]].

[5] L. Accardo et al. [AMS Collaboration], High Statistics Measurement of the Positron Fraction in Primary Cosmic Rays of 0.5?00 GeV with the Alpha Magnetic Spectrometer on the International Space Station, Phys. Rev. Lett. 113, 121101 (2014).

[6] M. Aguilar et al. [AMS Collaboration], Precision Measurement of the $\left(e^{+}+e^{-}\right)$Flux in Primary Cosmic Rays from $0.5 \mathrm{GeV}$ to $1 \mathrm{TeV}$ with the Alpha Magnetic Spectrometer on the International Space Station, Phys. Rev. Lett. 113, 221102 (2014).

[7] H. Yuksel, M. D. Kistler and T. Stanev, TeV Gamma Rays from Geminga and the Origin of the GeV Positron Excess, Phys. Rev. Lett. 103, 051101 (2009) [arXiv:0810.2784 [astro-ph]].

[8] D. Hooper, P. Blasi and P. D. Serpico, Pulsars as the Sources of High Energy Cosmic Ray Positrons, JCAP 0901, 025 (2009) [arXiv:0810.1527 [astro-ph]].

[9] S. Profumo, Dissecting cosmic-ray electron-positron data with Occam's Razor: the role of known Pulsars, Central Eur. J. Phys. 10, 1 (2011) [arXiv:0812.4457 [astro-ph]].

[10] D. Malyshev, I. Cholis and J. Gelfand, Pulsars versus Dark Matter Interpretation of ATIC/PAMELA, Phys. Rev. D 80, 063005 (2009) [arXiv:0903.1310 [astro-ph.HE]].

[11] H. B. Hu, Q. Yuan, B. Wang, C. Fan, J. L. Zhang and X. J. Bi, On the e+ e- excesses and the knee of the cosmic ray spectra - hints of cosmic rays acceleration in young supernova remnants, Astrophys. J. 700, L170 (2009) [arXiv:0901.1520 [astro-ph.HE]].

[12] P. Blasi, The origin of the positron excess in cosmic rays, Phys. Rev. Lett. 103, 051104 (2009) [arXiv:0903.2794 [astro-ph.HE]].

[13] T. Linden and S. Profumo, Probing the Pulsar Origin of the Anomalous Positron Fraction with AMS-02 and Atmospheric Cherenkov Telescopes, Astrophys. J. 772, 18 (2013) [arXiv:1304.1791 [astro-ph.HE]].

[14] P. F. Yin, Z. H. Yu, Q. Yuan and X. J. Bi, Pulsar interpretation for the AMS-02 result, Phys. Rev. D 88, no. 2, 023001 (2013) [arXiv:1304.4128 [astro-ph.HE]]. 
[15] L. Bergstrom, T. Bringmann and J. Edsjo, New Positron Spectral Features from Supersymmetric Dark Matter - a Way to Explain the PAMELA Data? Phys. Rev. D 78, 103520 (2008) [arXiv:0808.3725 [astro-ph]].

[16] V. Barger, W. Y. Keung, D. Marfatia and G. Shaughnessy, PAMELA and dark matter, Phys. Lett. B 672, 141 (2009) [arXiv:0809.0162 [hep-ph]].

[17] M. Cirelli, M. Kadastik, M. Raidal and A. Strumia, Model-independent implications of the e+-, anti-proton cosmic ray spectra on properties of Dark Matter, Nucl. Phys. B 813, 1 (2009) Addendum: [Nucl. Phys. B 873, 530 (2013)] [arXiv:0809.2409 [hep-ph]].

[18] P. f. Yin, Q. Yuan, J. Liu, J. Zhang, X. j. Bi and S. h. Zhu, PAMELA data and leptonically decaying dark matter, Phys. Rev. D 79, 023512 (2009) [arXiv:0811.0176 [hep-ph]].

[19] J. Zhang, X. J. Bi, J. Liu, S. M. Liu, P. F. Yin, Q. Yuan and S. H. Zhu, Discriminating different scenarios to account for the cosmic e $+/$ - excess by synchrotron and inverse Compton radiation, Phys. Rev. D 80, 023007 (2009) [arXiv:0812.0522 [astro-ph]].

[20] Sergio Palomares-Ruiz and Jennifer M. Siegal-Gaskins, Annihilation vs. Decay: Constraining dark matter properties from a gamma-ray detection, JCAP 1007, 023 (2010) [arXiv:1003.1142 [astro-ph]].

[21] L. Feng, R. Z. Yang, H. N. He, T. K. Dong, Y. Z. Fan and J. Chang, AMS-02 positron excess: new bounds on dark matter models and hint for primary electron spectrum hardening, Phys. Lett. B 728, 250 (2014) [arXiv:1303.0530 [astro-ph.HE]].

[22] K. Blum, B. Katz and E. Waxman, AMS-02 Results Support the Secondary Origin of Cosmic Ray Positrons, Phys. Rev. Lett. 111, no. 21, 211101 (2013) [arXiv:1305.1324 [astro-ph.HE]].

[23] L. Bergstrom, T. Bringmann, I. Cholis, D. Hooper and C. Weniger, New limits on dark matter annihilation from AMS cosmic ray positron data, Phys. Rev. Lett. 111, 171101 (2013) [arXiv:1306.3983 [astro-ph.HE]].

[24] J. Kopp, Constraints on dark matter annihilation from AMS-02 results, Phys. Rev. D 88, 076013 (2013) [arXiv:1304.1184 [hep-ph]].

[25] I. Cholis and D. Hooper, Dark Matter and Pulsar Origins of the Rising Cosmic Ray Positron Fraction in Light of New Data From AMS, Phys. Rev. D 88, 023013 (2013) [arXiv:1304.1840 [astro-ph.HE]].

[26] Q. Yuan, X. J. Bi, G. M. Chen, Y. Q. Guo, S. J. Lin and X. Zhang, Implications of the AMS-02 positron fraction in cosmic rays," Astropart. Phys. 60, 1 (2015) [arXiv:1304.1482 [astro-ph.HE]].

[27] H. B. Jin, Y. L. Wu and Y. F. Zhou, Implications of the first AMS-02 measurement for dark matter annihilation and decay, JCAP 1311, 026 (2013) [arXiv:1304.1997 [hep-ph]].

[28] P. S. Bhupal Dev, Dilip Kumar Ghosh, Nobuchika Okada and Ipsita Saha, Neutrino Mass and Dark Matter in light of recent AMS-02 results, Phys. Rev. D 89, 095001 (2014) [arXiv:1307.6204 [hep-ph]].

[29] K. Belotsky, M. Khlopov, C. Kouvaris and M. Laletin, High Energy Positrons and Gamma Radiation from Decaying Constituents of a two-component Dark Atom Model, Int.J.Mod.Phys. D24, no.13, 1545004 (2015) [arXiv:1508.02881 [astro-ph.HE]].

[30] J. Chang, Dark Matter Particle Explorer: The First Chinese Cosmic Ray and Hard Gamma-ray Detector in Space, Chinese Journal of Space Science 34, 550 (2014). 
[31] J. Chang et al. [DAMPE Collaboration], The DArk Matter Particle Explorer mission, Astropart. Phys. 95, 6 (2017) [arXiv:1706.08453 [astro-ph.IM]].

[32] G. Ambrosi et al. [DAMPE Collaboration], Direct detection of a break in the teraelectronvolt cosmic-ray spectrum of electrons and positrons, Nature 552, 63 (2017) [arXiv:1711.10981 [astro-ph.HE]].

[33] A. Fowlie, DAMPE squib? Significance of the 1.4 TeV DAMPE excess, Phys. Lett. B 780, 181 (2018) [arXiv:1712.05089 [hep-ph]].

[34] Q. Yuan et al., Interpretations of the DAMPE electron data, arXiv:1711.10989 [astro-ph.HE].

[35] L. Zu, C. Zhang, L. Feng, Q. Yuan and Y. Z. Fan, Constraints on the box-shaped cosmic ray electron feature from dark matter annihilation with the AMS-02 and DAMPE data, Phys. Rev. D 98, no. 6, 063010 (2018) [arXiv:1711.11052 [hep-ph]].

[36] Y. Z. Fan, W. C. Huang, M. Spinrath, Y. L. S. Tsai and Q. Yuan, A model explaining neutrino masses and the DAMPE cosmic ray electron excess, Phys. Lett. B 781, 83 (2018) [arXiv:1711.10995 [hep-ph]].

[37] H. B. Jin, B. Yue, X. Zhang and X. Chen, Dark matter explanation of the cosmic ray $e^{+} e^{-}$ spectrum excess and peak feature observed by the DAMPE experiment, Phys. Rev. D 98, no. 12, 123008 (2018) [arXiv:1712.00362 [astro-ph.HE]].

[38] P. H. Gu and X. G. He, Electrophilic dark matter with dark photon: from DAMPE to direct detection, Phys. Lett. B 778, 292 (2018) [arXiv:1711.11000 [hep-ph]].

[39] G. H. Duan, L. Feng, F. Wang, L. Wu, J. M. Yang and R. Zheng, Simplified TeV leptophilic dark matter in light of DAMPE data, JHEP 1802, 107 (2018) [arXiv:1711.11012 [hep-ph]].

[40] Y. L. Tang, L. Wu, M. Zhang and R. Zheng, Lepton-portal Dark Matter in Hidden Valley model and the DAMPE recent results, Sci. China Phys. Mech. Astron. 61, no. 10, 101003 (2018) [arXiv:1711.11058 [hep-ph]].

[41] W. Chao and Q. Yuan, The electron-flavored Z'-portal dark matter and the DAMPE cosmic ray excess, arXiv:1711.11182 [hep-ph].

[42] P. H. Gu, Radiative Dirac neutrino mass, DAMPE dark matter and leptogenesis, arXiv:1711.11333 [hep-ph].

[43] P. Athron, C. Balazs, A. Fowlie and Y. Zhang, Model-independent analysis of the DAMPE excess, JHEP 1802, 121 (2018) [arXiv:1711.11376 [hep-ph]].

[44] J. Cao, L. Feng, X. Guo, L. Shang, F. Wang and P. Wu, Scalar dark matter interpretation of the DAMPE data with U(1) gauge interactions, Phys. Rev. D 97, no. 9, 095011 (2018) [arXiv:1711.11452 [hep-ph]].

[45] G. H. Duan, X. G. He, L. Wu and J. M. Yang, Leptophilic dark matter in gauged $U(1)_{L_{e}-L_{\mu}}$ model in light of DAMPE cosmic ray $e^{+}+e^{-}$excess, Eur. Phys. J. C 78, no. 4, 323 (2018) [arXiv:1711.11563 [hep-ph]].

[46] X. Liu and Z. Liu, TeV dark matter and the DAMPE electron excess, Phys. Rev. D 98, no. 3, 035025 (2018) [arXiv:1711.11579 [hep-ph]].

[47] X. J. Huang, Y. L. Wu, W. H. Zhang and Y. F. Zhou, Origins of sharp cosmic-ray electron structures and the DAMPE excess, Phys. Rev. D 97, 091701 (2018) [arXiv:1712.00005 [astro-ph.HE]]. 
[48] W. Chao, H. K. Guo, H. L. Li and J. Shu, Electron Flavored Dark Matter, Phys. Lett. B 782, 517 (2018) [arXiv:1712.00037 [hep-ph]].

[49] Y. Gao and Y. Z. Ma, Dark matter cascade decay implications from DAMPE, HESS, Fermi-LAT and AMS02, arXiv:1712.00370 [astro-ph.HE].

[50] J. S. Niu, T. Li, R. Ding, B. Zhu, H. F. Xue and Y. Wang, Bayesian analysis of the break in $D A M P E$ lepton spectra, Phys. Rev. D 97, no. 8, 083012 (2018) [arXiv:1712.00372 [astro-ph.HE]].

[51] C. H. Chen, C. W. Chiang and T. Nomura, Explaining the DAMPE $e^{+} e^{-}$excess using the Higgs triplet model with a vector dark matter, Phys. Rev. D 97, no. 6, 061302 (2018) [arXiv:1712.00793 [hep-ph]].

[52] T. Li, N. Okada and Q. Shafi, Scalar dark matter, Type II Seesaw and the DAMPE cosmic ray $e^{+}+e^{-}$excess, Phys. Lett. B 779, 130 (2018) [arXiv:1712.00869 [hep-ph]].

[53] R. Zhu and Y. Zhang, Graviton-mediated dark matter model explanation the DAMPE electron excess and search at $e^{+} e^{-}$colliders, arXiv:1712.01143 [hep-ph].

[54] P. H. Gu, Quasi-degenerate dark matter for DAMPE excess and $3.5 \mathrm{keV}$ line, Sci. China Phys. Mech. Astron. 61, no. 10, 101005 (2018) [arXiv:1712.00922 [hep-ph]].

[55] T. Nomura and H. Okada, Radiative seesaw models linking to dark matter candidates inspired by the DAMPE excess, Phys. Dark Univ. 21, 90 (2018) [arXiv:1712.00941 [hep-ph]].

[56] K. Ghorbani and P. H. Ghorbani, DAMPE electron-positron excess in leptophilic Z' model, JHEP 1805, 125 (2018) [arXiv:1712.01239 [hep-ph]].

[57] J. Cao, L. Feng, X. Guo, L. Shang, F. Wang, P. Wu and L. Zu, Explaining the DAMPE data with scalar dark matter and gauged $U(1)_{L_{e}-L_{\mu}}$ interaction, Eur. Phys. J. C 78, no. 3, 198 (2018) [arXiv:1712.01244 [hep-ph]].

[58] F. Yang and M. Su, Dark Matter Annihilation from Nearby Ultra-compact Micro Halos to Explain the Tentative Excess at $1.4 \mathrm{TeV}$ in DAMPE data, arXiv:1712.01724 [astro-ph.HE].

[59] R. Ding, Z. L. Han, L. Feng and B. Zhu, Confronting the DAMPE Excess with the Scotogenic Type-II Seesaw Model, Chin. Phys. C 42, no. 8, 083104 (2018) [arXiv:1712.02021 [hep-ph]].

[60] G. L. Liu, F. Wang, W. Wang and J. M. Yang, Explaining DAMPE results by dark matter with hierarchical lepton-specific Yukawa interactions, Chin. Phys. C 42, no. 3, 035101 (2018) [arXiv:1712.02381 [hep-ph]].

[61] S. F. Ge, H. J. He and Y. C. Wang, Flavor Structure of the Cosmic-Ray Electron/Positron Excesses at DAMPE, Phys. Lett. B 781, 88 (2018) [arXiv:1712.02744 [astro-ph.HE]].

[62] Y. Zhao, K. Fang, M. Su and M. C. Miller, A strong test of the dark matter origin of a TeV electron excess using icecube neutrinos, JCAP 1806, no. 06, 030 (2018) [arXiv:1712.03210 [astro-ph.HE]].

[63] Y. Sui and Y. Zhang, Prospects of type-II seesaw models at future colliders in light of the DAMPE $e^{+} e^{-}$excess, Phys. Rev. D 97, no. 9, 095002 (2018) [arXiv:1712.03642 [hep-ph]].

[64] N. Okada and O. Seto, DAMPE excess from decaying right-handed neutrino dark matter, Mod. Phys. Lett. A 33, no. 27, 1850157 (2018) [arXiv:1712.03652 [hep-ph]].

[65] J. Cao, X. Guo, L. Shang, F. Wang, P. Wu and L. Zu, Scalar dark matter explanation of the DAMPE data in the minimal Left-Right symmetric model, Phys. Rev. D 97, no. 6, 063016 (2018) [arXiv:1712.05351 [hep-ph]]. 
[66] Z. L. Han, W. Wang and R. Ding, Radiative Seesaw Model and DAMPE Excess from Leptophilic Gauge Symmetry, Eur. Phys. J. C 78, no. 3, 216 (2018) [arXiv:1712.05722 [hep-ph]].

[67] J. S. Niu, T. Li and F. Z. Xu, A Simple and Natural Interpretations of the DAMPE Cosmic Ray Electron/Positron Spectrum within Two Sigma Deviations, Eur. Phys. J. C 79, no. 2, 125 (2019) [arXiv:1712.09586 [hep-ph]].

[68] T. Nomura, H. Okada and P. Wu, A radiative neutrino mass model in light of DAMPE excess with hidden gauged $U(1)$ symmetry, JCAP 1805, no. 05, 053 (2018) [arXiv:1801.04729 [hep-ph]].

[69] B. Wang, X. Bi, S. Lin and P. Yin, Explanations of the DAMPE high energy electron/positron spectrum in the dark matter annihilation and pulsar scenarios, Sci. China Phys. Mech. Astron. 61, no. 10, 101004 (2018).

[70] X. Pan, C. Zhang and L. Feng, Interpretation of the DAMPE 1.4 TeV peak according to the decaying dark matter model, Sci. China Phys. Mech. Astron. 61, no. 10, 101006 (2018).

[71] X. Liu, Z. Liu and Y. Su, Two-mediator dark matter models and cosmic electron excess, arXiv:1902.04916 [hep-ph].

[72] Q. Yuan and L. Feng, Dark Matter Particle Explorer observations of high-energy cosmic ray electrons plus positrons and their physical implications, Sci. China Phys. Mech. Astron. 61, no. 10, 101002 (2018) [arXiv:1807.11638 [astro-ph.HE]].

[73] C. R. Chen, S. K. Mandal and F. Takahashi, Gamma-ray Constraints on Hadronic and Leptonic Activities of Decaying Dark Matter, JCAP 1001, 023 (2010) [arXiv:0910.2639 [hep-ph]].

[74] G. Hutsi, A. Hektor and M. Raidal, Implications of the Fermi-LAT diffuse gamma-ray measurements on annihilating or decaying Dark Matter, JCAP 1007, 008 (2010) [arXiv:1004.2036 [astro-ph.HE]].

[75] M. Cirelli, E. Moulin, P. Panci, P. D. Serpico and A. Viana, Gamma ray constraints on Decaying Dark Matter, Phys. Rev. D 86, 083506 (2012) [arXiv:1205.5283 [astro-ph.CO]].

[76] H. C. Cheng, W. C. Huang, X. Huang, I. Low, Y. L. S. Tsai and Q. Yuan, AMS-02 Positron Excess and Indirect Detection of Three-body Decaying Dark Matter, JCAP 1703, no. 03, 041 (2017) [arXiv:1608.06382 [hep-ph]].

[77] P. A. R. Ade et al. [Planck Collaboration], Planck 2015 results. XIII. Cosmological parameters, Astron. Astrophys. 594, A13 (2016) [arXiv:1502.01589 [astro-ph.CO]].

[78] A. W. Strong, I. V. Moskalenko and V. S. Ptuskin, Cosmic-ray propagation and interactions in the Galaxy, Ann. Rev. Nucl. Part. Sci. 57, 285 (2007) [astro-ph/0701517].

[79] Q. Yuan, S. J. Lin, K. Fang and X. J. Bi, Propagation of cosmic rays in the AMS-02 era, Phys. Rev. D 95, no. 8, 083007 (2017) [arXiv:1701.06149 [astro-ph.HE]].

[80] Q. Yuan, Implications on cosmic ray injection and propagation parameters from Voyager/ACE/AMS-02 nucleus data, Sci. China Phys. Mech. Astron. 62, no. 4, 49511 (2019) [arXiv:1805.10649 [astro-ph.HE]].

[81] M. Ackermann et al. [Fermi-LAT Collaboration], Constraints on the Galactic Halo Dark Matter from Fermi-LAT Diffuse Measurements, Astrophys. J. 761, 91 (2012) [arXiv:1205.6474 [astro-ph.CO]]. 
[82] M. Ackermann et al. [Fermi-LAT Collaboration], Fermi-LAT Observations of the Diffuse Gamma-Ray Emission: Implications for Cosmic Rays and the Interstellar Medium, Astrophys. J. 750, 3 (2012) [arXiv:1202.4039 [astro-ph.HE]].

[83] A. W. Strong and I. V. Moskalenko, Propagation of cosmic-ray nucleons in the galaxy, Astrophys. J. 509, 212 (1998) [astro-ph/9807150].

[84] C. Evoli, D. Gaggero, D. Grasso and L. Maccione, Cosmic-Ray Nuclei, Antiprotons and Gamma-rays in the Galaxy: a New Diffusion Model, JCAP 0810, 018 (2008) Erratum: [JCAP 1604, no. 04, E01 (2016)] [arXiv:0807.4730 [astro-ph]].

[85] X. Huang, Y. L. S. Tsai and Q. Yuan, LikeDM: likelihood calculator of dark matter detection, Comput. Phys. Commun. 213, 252 (2017) [arXiv:1603.07119 [hep-ph]].

[86] A. M. Atoyan, F. A. Aharonian and H. J. Volk, Electrons and positrons in the galactic cosmic rays, Phys. Rev. D 52, 3265 (1995).

[87] J. N. Bahcall and R. M. Soneira, The Universe at faint magnetidues. I. Models for the galaxy and the predicted star counts, Astrophys. J. Suppl. 44, 73 (1980).

[88] J. F. Navarro, C. S. Frenk and S. D. M. White, A Universal density profile from hierarchical clustering, Astrophys. J. 490, 493 (1997) [astro-ph/9611107].

[89] V. Springel et al., The Aquarius Project: the subhalos of galactic halos, Mon. Not. Roy. Astron. Soc. 391, 1685 (2008) [arXiv:0809.0898 [astro-ph]].

[90] A. Coogan, B. V. Lehmann, S. Profumo, Connecting direct and indirect detection with a dark spike in the cosmic-ray electron spectrum, JCAP 1910, 063 (2019) [arXiv:1903.07177 [astro-ph.HE]].

[91] S. F. Ge, H. J. He, Y. C. Wang and Q. Yuan, in preparation.

[92] Y. Zhao, X. J. Bi, S. J. Lin and P. F. Yin, Nearby dark matter subhalo that accounts for the DAMPE excess, Chinese Physics C Vol. 43, No. 8 (2019) 085101.

[93] P. Agrawal, Z. Chacko and C. B. Verhaaren, Leptophilic Dark Matter and the Anomalous Magnetic Moment of the Muon, JHEP 1408, 147 (2014) [arXiv:1402.7369 [hep-ph]].

[94] S. L. Chen and Z. Kang, JCAP 1805, 036 (2018).

[95] C. M. Ho and R. J. Scherrer, Phys. Lett. B 722, 341 (2013).

[96] A. Birkedal, A. Noble, M. Perelstein and A. Spray, Little Higgs dark matter, Phys. Rev. D 74, 035002 (2006) [hep-ph/0603077].

[97] G. Servant and T. M. P. Tait, Is the lightest Kaluza-Klein particle a viable dark matter candidate? Nucl. Phys. B 650, 391 (2003) [hep-ph/0206071].

[98] H. C. Cheng, J. L. Feng and K. T. Matchev, Kaluza-Klein dark matter, Phys. Rev. Lett. 89, 211301 (2002) [hep-ph/0207125].

[99] C. T. Hill and R. J. Hill, $T^{-}$parity violation by anomalies, Phys. Rev. D 76, 115014 (2007) [arXiv:0705.0697 [hep-ph]].

[100] S. Baek and Z. F. Kang, Naturally Large Radiative Lepton Flavor Violating Higgs Decay Mediated by Lepton-flavored Dark Matter, JHEP 1603, 106 (2016) [arXiv:1510.00100 [hep-ph]].

[101] L. J. Gleeson and W. I. Axford, Solar Modulation of Galactic Cosmic Rays, Astrophys. J. 154, 1011 (1968). 